\title{
Thermodynamics and Kinetics in Antibody Resistance of the 501Y.V2 SARS-CoV-2 Variant
}

\author{
Son Tung Ngo, ${ }^{\text {ab }}$ Trung Hai Nguyen, ${ }^{\text {ab }}$ Duc-Hung Pham, ${ }^{c}$ Nguyen Thanh Tung, ${ }^{\text {de }}$ and Pham Cam Nam ${ }^{f *}$ \\ aLaboratory of Theoretical and Computational Biophysics, Ton Duc Thang University, Ho Chi Minh City, Vietnam; Email: ngosontung@tdtu.edu.vn \\ bFaculty of Applied Sciences, Ton Duc Thang University, Ho Chi Minh City, Vietnam \\ 'Division of Immunobiology, Hepatology and Nutrition Cincinnati Children's Hospital Medical Center, Cincinnati 45229, OH, USA \\ dInstitute of Materials Science, Vietnam Academy of Science and Technology, Hanoi, Vietnam \\ eGraduate University of Science and Technology, Vietnam Academy of Science and Technology, Hanoi, Vietnam

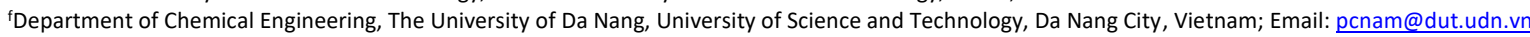

In this context, the South African variant (B1.351 or 501Y.V2) can resist the neutralizing antibody (NAb). Three substitutions in RBD including K417N, E484K, and N501Y alters the free energy landscape, binding pose, binding free energy, binding kinetics, and unbinding pathway of RBD + NAb complexes.

The novel $\beta$-coronavirus, SARS-CoV-2, whose sequence is similar to SARS-CoV- 1 and MERS-CoV inducing human respiratory epidemic in the beginning of this century, is the cause of the human respiratory disease (COVID-19) pandemic worldwide. ${ }^{1}, 2$ This virus has been infecting more than 100 million cases and associated with more than 2 million deaths. SARS-CoV-2 is a single-positive-strand RNA virus, whose genome encodes for four main components: spike, envelope, membrane and nucleocapsid. ${ }^{3,} 4$ The spike protein (protein S) of SARS-CoV-2 which is used by the virus to bind to human angiotensinconverting-enzyme 2 (ACE2), has been researched thoroughly. ACE2 is present in different tissues in the body, including the lung, heart and liver, ${ }^{5}$ is employed by SARS-CoV-2 as receptor to bind and infect human cells. The $S$ trimer comprises three copies of $S 1$ and S2 subunits. S1 subunit contains 4 domains: S1A, S1B, S1C and S1D, in which S1B domain is also called receptor-binding domain (RBD), which mediates the attachment of spike protein to target cell via binding to ACE2 receptor. ${ }^{6}$ Once RBD is in the 'up' conformation, it can recognize and bind into ACE2, which leads to the conformational changes of S2 subunit and enables SARS-CoV-2 to fuse with cell membrane and to enter host cells. ${ }^{1,6}$

RBD is the main target of neutralizing antibodies (NAbs) which can be isolated from plasma of COVID-19 patients, immunoglobulin libraries, or immunized laboratory animal models ${ }^{1}$. These NAbs can be roughly divided into four main classes, of which class $1 \mathrm{~s}^{\prime}$ and class $2 s^{\prime}$ RBD epitopes overlap with the ACE2-binding site, suggesting a neutralization mechanism that involves direct competition with ACE2. Class 1 antibodies, which are encoded by the immunoglobulin V-gene (VH3-53) segment with complementarity-determining regions 1 and 2 (CDRH1 and $\mathrm{CDRH} 2$ ) and a short $\mathrm{CDRH} 3$, are mostly elicited by SARS-CoV-2 infection. On the other hand, when class 2 antibodies also target site 110,15 which is also target epitopes of class 1 antibodies, they bind to RBD in both 'up' and down' conformations of $S$ protein. ${ }^{1,7}$ Additionally, class 3 antibodies bind outside ACE2 and recognize both up and down RBD, while class 4 antibodies comprise previously described antibodies that cannot block ACE2 and target only to RBD in 'up' conformation. ${ }^{1}$ Besides RBD, the N-terminal domain (NTD) of protein $\mathrm{S}$ is also a popular target for NAbs and many potent monoclonal antibodies directed against this region show great potential in clinical trials for COVID-19 treatment. ${ }^{7}$ The majority of these antibodies target a single immunodominant site on NTD, including the N1-loop (NTD N-terminus), N3-loop (supersite bhairpin), and N5 loop (supersite loop). Subsets of these antibodies and NAbs in class 1 and class 3 form multi-donor classes, with different set of $\mathrm{VH}$ germline restricted mode of spike recognition. ${ }^{7}$

Due to many reasons, including high transmissibility, the longevity of the pandemic, and encountering with immunocompromised hosts, SARS-CoV-2 undergoes different rounds of mutations, which has altered the structures of the virus, modulated its infectivity, and changed the antigenicity of the surface proteins. ${ }^{8}$ The variants, including United Kingdom (B1.1.7) and South African (B1.351 or 501Y.V2) variants have associated with increased transmissibility and possibly increased mortality. ${ }^{7}$ Especially, the SARS-CoV-2 lineage in South Africa, included nine mutations in the spike protein, seems to decrease the efficacy of NAb as well as Covid-19 vaccine efficacy of some vaccines currently being used..$^{9,10}$ The mutations in B1.351 can be divided into two groups, one concentrates in NTD, including four substitutions and a deletion (L18F, D80A, D215G, $2242-244$, and $\mathrm{R} 246 \mathrm{I})$, and the other involves three substitutions in RBD (K417N, E484K, and N501Y). ${ }^{11}$

Evaluating antibody resistance of the 501Y.V2 SARS-CoV-2 variant is greatly attractive to scientists. $7,9,10,12$ Understanding the physical insights into the process probably enhances the vaccine developments, but the knowledge is still limited. Therefore, in this context, atomistic simulations were carried out to reveal the insights at the atomic level of the binding process of NAb and fragment NAb (fNAb) to 501Y.V2 SARS-CoV-2 RBD. Structural changes of the WT/501Y.V2 SARS-CoV-2 RBD + fNAb/NAb complexes were characterized via unbiased MD simulations. Thermodynamics and kinetics of the binding process were then revealed via biased MD simulations. Details of simulations were described in Figure 1 and the Electronic Supplementary (ESI) file. In particular, GROMCS 5.1.5 13 was used to investigate, whereas Amber99SB-iLDN force field ${ }^{14}$ and TIP3P water model 15 were used to represent protein and water molecules, respectively. 
(A)
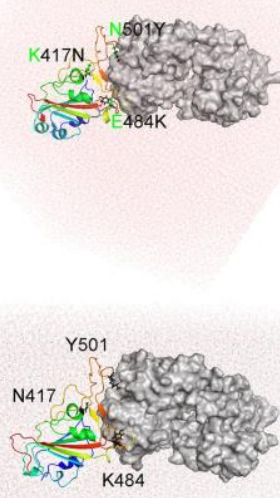

(B)
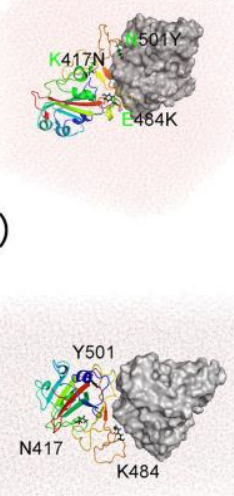

(D)

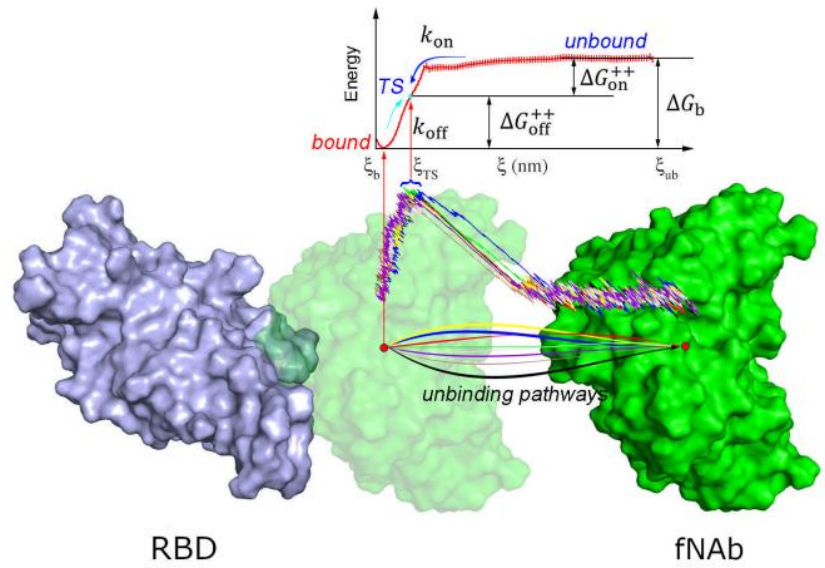

(E)

(C)

Figure 1. Starting structures of RBD + NAb systems. (A) WT/501Y.V2 RBD + NAb in MD simulations; (B) WT/501Y.V2 RBD + fNAb in MD simulations; (C) + (D) 501Y.V2 RBD + NAb/fNAb in SMD/US simulations; (E) Free energy scheme. The fNAb was mobilized from bound to unbound states via FPL calculations, then the free energy profile was calculated via US simulations.

Unbiased MD simulations were carried out to understand the structural change at the atomistic level of 501Y.V2 RBD + NAbs since the binding affinity of the NAbs to 501Y.V2 RBD was altered according to the recent report.7, 9, 10, 12 The stabilized conformations of the RBD + NAb complexes were investigated over the equilibrium trajectories (cf. Figure S1 of the ESI file). The principal component analysis (PCA) method was employed to generate the free energy landscape (FEL) of RBD + NAb systems. ${ }^{16}$ The obtained results were described in Figure 2 . Clearly, the 501Y.V2 variant increases the number of the FEL local minima implying that the 501Y.V2 complex is more flexible than the WT one. It also shows that the binding free energy $\Delta G_{\mathrm{b}}$ between 501Y.V2 RBD and NAbs is significantly reduced.
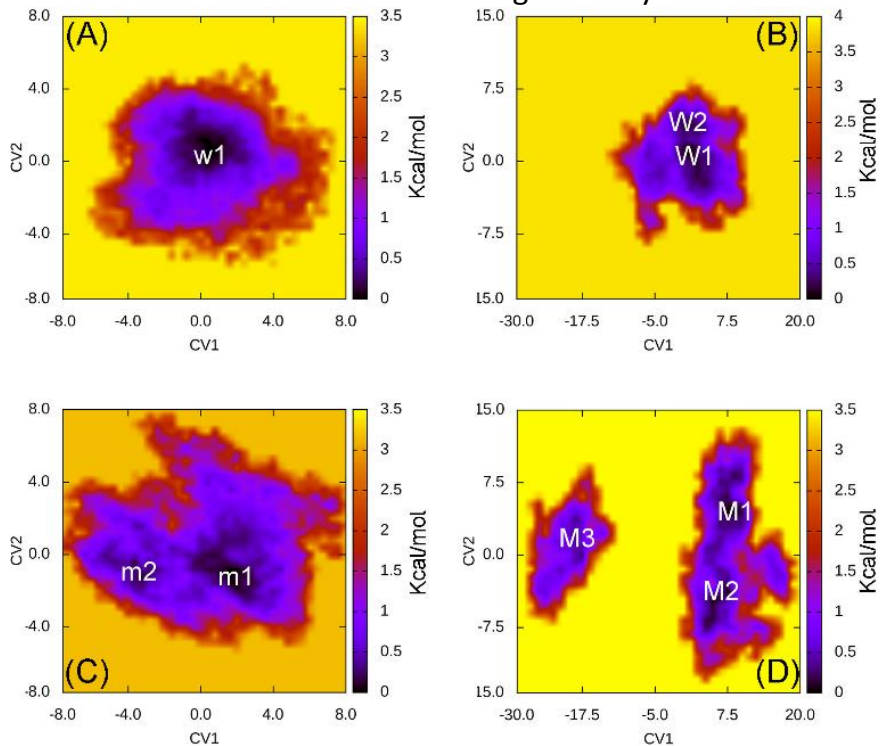

Figure 2. Free energy landscape of RBD + antibody was constructed using PCA method. In particular, (A) presents the FEL of the WT RBD + fNAb; (B) mentions the FEL of the WT
RBD + NAb; (C) describes the FEL of the 501Y.V2 RBD + fNAb; and (D) denotes the FEL of the 501Y.V2 RBD + NAb.

The WT RBD + fNAb only formed one minimum noted as w1 in Figure 2A, which is located at (CV1; CV2) coordinates of $(0.40$; 0.40). In particular, the antibody adopted HBs to 4 residues of the WT RBD including G447, Y449, N450, and E484 (cf. Figure 3). These results suggest that a mutation E484K will be significantly altered the binding affinity/mechanism of the RBD + fNAb. Two minima were observed in FEL of 501Y.V2 RBD + fNAb, which are located at (CV1; CV2) coordinates of (1.60; $1.40)$ and $(-3.60 ;-1.00)$ denoted as $\mathbf{m} \mathbf{1}$ and $\mathbf{m} \mathbf{2}$, respectively. Analyzing the representative structure $\mathbf{m} \mathbf{1}$, the antibody was found to be able to form HBs to the residues K444, G447, Y449, and N450 of the 501Y.V2 RBD. The corresponding residues of m2, which formed HBs to RBD 2-4, are G447, Y449, N450, and K484 (cf. Figure 3). The observed structural changes imply that the binding affinity and kinetics between RBD and fNAb probably change. 


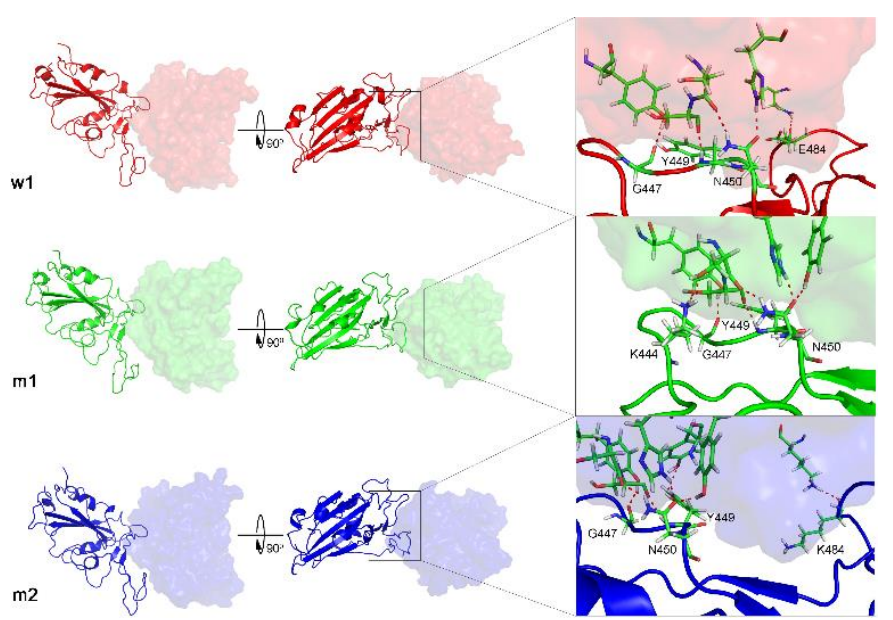

Figure 3. The representative structures of WT and 501Y.V2 RBD + fNAb in different perspective. The structures corresponds to the minima $\mathbf{w} \mathbf{1}, \mathbf{m} \mathbf{1}$, and $\mathbf{m} \mathbf{2}$.

The WT/501Y.V2 RBD + NAb systems were also investigated. FEL of the complexes was significantly altered when the mutations were induced. The WT RBD + NAb formed two minima, which were shown in Figure 2B. These minima located at (CV1; CV2) coordinates of $(0.63 ; 0.75)$ and $(0.63 ; 3.38)$ denoting as $\mathbf{W} \mathbf{1}$ and $\mathbf{W} \mathbf{2}$, respectively. Besides that, the 501Y.V2 $\mathrm{RBD}+\mathrm{NAb}$ FEL (Figure 2D) adopted three minima, which located at (CV1; CV2) coordinates of (7.50; 4.13), (5.63; -4.50), and $(-18.8 ; 1.50)$ labelling as $\mathbf{M} 1, \mathbf{M} 2$, and $\mathbf{M} 3$, respectively. Analyzing the complex $\mathbf{W} \mathbf{1}$, the HBs were observed between antibody and residues G447, Y449, N450, and E484 of the RBD that is in good consistency to the w1 case. However, HBs were only found between the NAb and residue E484 of the WT RBD in the complex W2 (Figure 4). The obtained results indicate that residue E484 plays an important role in the binding process of the antibody to the RBD. Replacing the E484 with another residue probably modifies the binding mechanism of the NAb to RBD rather than substitutions at the different positions. Moreover, it should be noted that in the 501Y.V2 variant induced, a lysine residue substitutes the glutamate residue at the sequence 484 . The replacement probably terminates the $\mathrm{HBs}$ and weakening the attracted force between the NAb and the RBD. The argument was confirmed via evaluations of the representative structures of 501Y.V2 RBD + NAb complexes. In conformation M1, the HBs between NAb and the residues $\mathrm{G} 447$ Y449, and N450 of RBD were found. The residues G447, Y449, $\mathrm{N} 450$, and T470 of 501Y.V2 RBD procedure HBs to NAb in conformation M2. Furthermore, The NAb only found two HBs to the residue E471 and N481 of the 501Y.V2 RBD. The free energy approach should be carried out to clarify the change of binding affinity upon the structural changes of the 501Y.V2 RBD + NAb complexes. As discussed above, the RBD + fNAb structure is more flexible when the 501Y.V2 variant was induced. The binding affinity/mechanism of the complex is thus altered. In this work, a combination of steered-molecular dynamics (SMD)/umbrella sampling (US) simulations were carried out to probe the change in RBD + NAbs association. The SMD was used to generate US windows (cf. the ESI file). The free energy profile was then calculated using the WHAM. ${ }^{17}$ The binding free energy $\Delta G_{\mathrm{b}}$ between RBD and NAbs is able to calculate via PMF curve as mentioned in Figure 1E. ${ }^{18,}{ }^{19}$
Moreover, the free energy barriers $\Delta G_{\text {on }}^{++}$and $\Delta G_{\text {off }}^{++}$, which were associated with the binding kinetic rate constant $k_{\text {on }}$ and the unbinding kinetic rate constant $k_{\text {off }}$ can be also estimated, respectively.

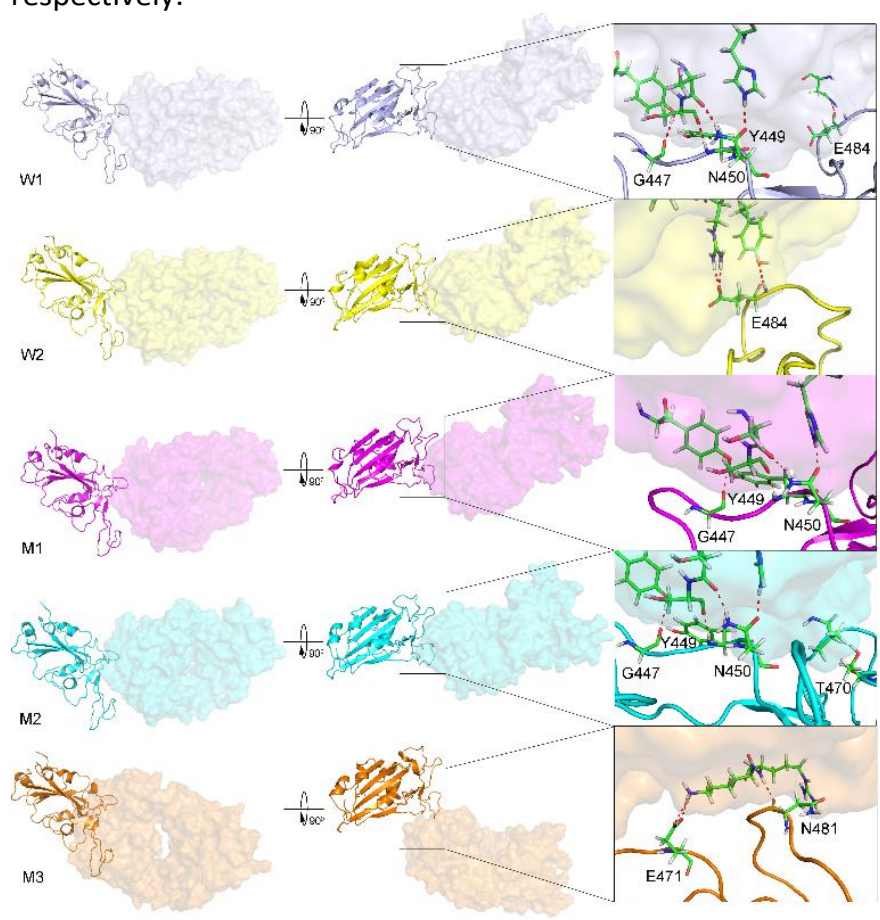

Figure 4. The representative structures of WT and 501Y.V2 RBD + NAb corresponding to the minima W1, W2, M1, M2, and $\mathbf{M} 3$. The interaction diagram between RBD and NAb were obtained using PyMOL tool.

The calculated results for free energy barriers (cf. Table S2) indicated that the NAbs will bind to 501Y.V2 RBD more difficult than WT one because of the larger $\Delta G_{\text {on }}^{++}$.NAbs are much easier to bind to than to unbind from RBD, because the $\Delta G_{\text {off }}^{++}$is larger than the $\Delta G_{\text {on }}^{++}$. However, in the M3 case, the $\Delta G_{\text {off }}^{++}=0.14 \pm$ $0.18 \mathrm{kcal} \mathrm{mol}^{-1}$ is significantly smaller than the $\Delta G_{\text {on }}^{++}=2.83 \pm$ $0.65 \mathrm{kcal} \mathrm{mol}^{-1}$ indicating that it takes more time for NAb to bind to 501Y.V2 RBD for them unbind. Moreover, the observations were also confirmed due to the binding free energy, $\Delta G_{\mathrm{b}}$, calculations, in which the thermodynamic metric corresponding to the association between NAbs and RBD is significantly decreased when the 501Y.V2 variant was induced (Table S2). The NAb is thus resisted to bind to 501Y.V2 RBD. Therefore, it may be argued that the 501Y.V2 variant could reduce the vaccine efficiency. The observation is in good agreement with the experimental data. 7, 9, 10,12

In addition, the collective-variable $\mathrm{FEL},{ }^{20}$ was constructed by number of contacts between two proteins within $0.45 \mathrm{~nm}$ and the displacement of the antibody, revealed the unbinding pathway of NAbs. The obtained FEL was shown in Figure $\mathbf{5}$ and Figure S2 of the ESI file. The representative structures of the complexes within a backbone RMSD of $0.2 \mathrm{~nm}$ were then estimated using clustering method. ${ }^{16}$ The unbinding pathways were significantly altered under effects of the 501Y.V2 variant. A larger number of transition states of the WT RBD + fNAb complex implies that it is hard to unbind the antibody from WT system than 501Y.V2 variant. Moreover, the representative structures $\mathbf{B}, \mathbf{b}$, and $\mathbf{b}^{\prime}$ correspond to the binding model of the $R B D+f N A b$ complexes. The structures $\mathbf{D 7}, \mathbf{d 6}$, and $\mathbf{d 4} \mathbf{4}^{\prime}$ respond 
to the minima where the fNAb completely detached from RBD. The other conformations correspond to dissociated structures along unbinding pathways. The similar picture was also observed when the RBD + NAb complexes were investigated (Figure S2).

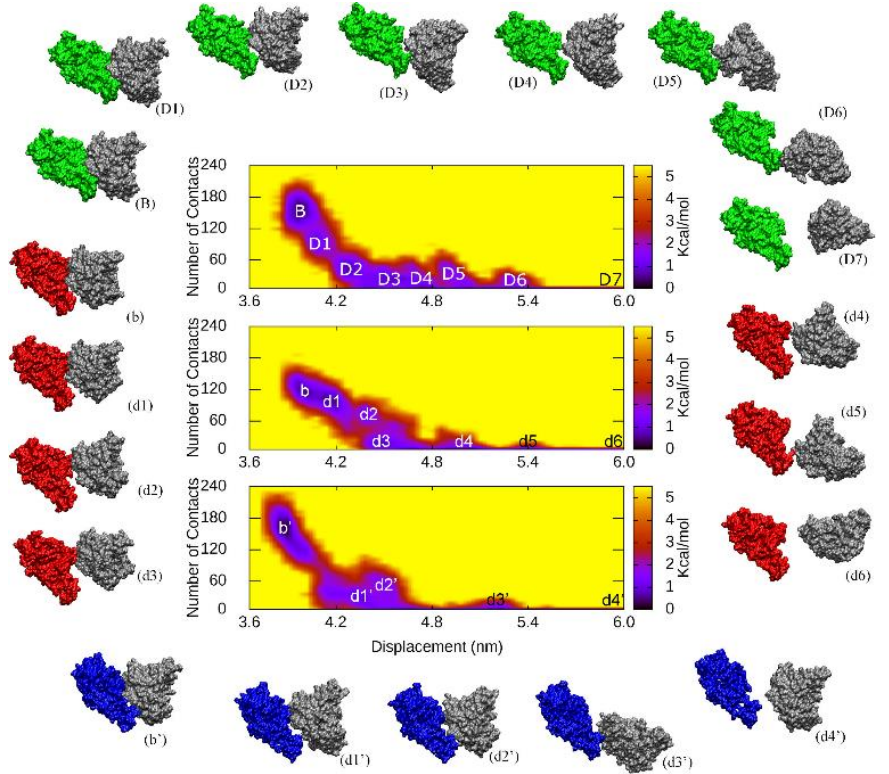

Figure 5. The collective-variable FEL revealed the unbinding pathways of fNAb from the binding mode with WT/501Y.V2 RBD. The representative structures of complexes were also estimated.

In this work, the NAb resistance of 501Y.V2 variant was revealed at atomic level using biased and unbiased MD simulations. In particular, the binding pose of NAb/fNAb to WT/501Y.V2 RBD was revealed using atomistic simulations. Increasing FEL minima of 501Y.V2 RBD + NAb/fNAb in comparison with the WT RBD systems infer that the complex 501Y.V2 RBD + NAb/fNAb is more unstable than the WT one. Thermodynamics and kinetics of the binding process between RBD and NAb were also determined using SMD/US simulations. Interestingly, the binding free energy $\Delta G_{\mathrm{b}}$ of WT RBD + NAb/fNAb is significantly smaller than that of 501Y.V2 RBD + $\mathrm{NAb} / \mathrm{fNAb}$. It is consistent with the results of the binding kinetic rate constant $k_{\text {on }}$ and the unbinding kinetic rate constant $k_{\text {off }}$. Poorly binding affinity of NAb/fNAb to 501Y.V2 RBD confirm the antibody resistance of the South African variant. ${ }^{7,9,10,12}$

\section{Author Contributions}

All authors contributed to doing computations and preparing the manuscript.

\section{Conflicts of interest}

There are no conflicts to declare.

\section{Notes and references}

C. O. Barnes, A. P. West, K. E. Huey-Tubman, M. A. G. Hoffmann, N. G. Sharaf, P. R. Hoffman, N. Koranda, H. B. Gristick, C. Gaebler, F. Muecksch, J. C. C. Lorenzi, S. Finkin, T. Hägglöf, A. Hurley, K. G. Millard, Y. Weisblum, F. Schmidt,
T. Hatziioannou, P. D. Bieniasz, M. Caskey, D. F. Robbiani, M. C. Nussenzweig and P. J. Bjorkman, Cell, 2020, 182, 828842.e816

2. E. de Wit, N. van Doremalen, D. Falzarano and V. J. Munster, Nature Reviews Microbiology, 2016, 14, 523-534.

3. S. T. Ngo, N. Quynh Anh Pham, L. Thi Le, D.-H. Pham and V. V. Vu, Journal of Chemical Information and Modeling, 2020, 60, 5771-5780.

4. D. Schoeman and B. C. Fielding, Virology Journal, 2019, 16, 69.

5. M. Hoffmann, H. Kleine-Weber, S. Schroeder, N. Kruger, T. Herrler, S. Erichsen, T. S. Schiergens, G. Herrler, N. H. Wu, A. Nitsche, M. A. Muller, C. Drosten and S. Pohlmann, Cell, 2020, DOI: 10.1016/j.cell.2020.02.052.

6. J. Lan, J. Ge, J. Yu, S. Shan, H. Zhou, S. Fan, Q. Zhang, X. Shi, Q. Wang, L. Zhang and X. Wang, Nature, 2020, 581, 215220.

C. K. Wibmer, F. Ayres, T. Hermanus, M. Madzivhandila, P. Kgagudi, B. Oosthuysen, B. E. Lambson, T. de Oliveira, M. Vermeulen, K. van der Berg, T. Rossouw, M. Boswell, V. Ueckermann, S. Meiring, A. von Gottberg, C. Cohen, L. Morris, J. N. Bhiman and P. L. Moore, Nature Medicine, 2021, DOI: 10.1038/s41591-021-01285-x.

WHO, 2020.

P. Wang, M. S. Nair, L. Liu, S. Iketani, Y. Luo, Y. Guo, M. Wang, J. Yu, B. Zhang, P. D. Kwong, B. S. Graham, J. R. Mascola, J. Y. Chang, M. T. Yin, M. Sobieszczyk, C. A. Kyratsous, L. Shapiro, Z. Sheng, Y. Huang and D. D. Ho, Nature, 2021, DOI: 10.1038/s41586-021-03398-2.

M. Hoffmann, P. Arora, R. Groß, A. Seidel, B. F. Hörnich, A. S. Hahn, N. Krüger, L. Graichen, H. Hofmann-Winkler, A. Kempf, M. S. Winkler, S. Schulz, H.-M. Jäck, B. Jahrsdörfer, H. Schrezenmeier, M. Müller, A. Kleger, J. Münch and S. Pöhlmann, Cell, 2021, DOI: 10.1016/j.cell.2021.03.036.

11. Y. Weisblum, F. Schmidt, F. Zhang, J. DaSilva, D. Poston, J. C. C. Lorenzi, F. Muecksch, M. Rutkowska, H.-H. Hoffmann, E. Michailidis, C. Gaebler, M. Agudelo, A. Cho, Z. Wang, A. Gazumyan, M. Cipolla, L. Luchsinger, C. D. Hillyer, M. Caskey, D. F. Robbiani, C. M. Rice, M. C. Nussenzweig, T. Hatziioannou and P. D. Bieniasz, eLife, 2020, 9, e61312.

12. S. Cele, I. Gazy, L. Jackson, S.-H. Hwa, H. Tegally, G. Lustig, J. Giandhari, S. Pillay, E. Wilkinson, Y. Naidoo, F. Karim, Y. Ganga, K. Khan, M. Bernstein, A. B. Balazs, B. I. Gosnell, W. Hanekom, M.-Y. S. Moosa, R. J. Lessells, T. de Oliveira, A. Sigal, S. A. Ngs and C.-K. Team, Nature, 2021, DOI: 10.1038/s41586-021-03471-w.

13. M. J. Abraham, T. Murtola, R. Schulz, S. Páll, J. C. Smith, B. Hess and E. Lindahl, SoftwareX, 2015, 1-2, 19-25.

14. A. E. Aliev, M. Kulke, H. S. Khaneja, V. Chudasama, T. D. Sheppard and R. M. Lanigan, Proteins: Struct., Funct., Bioinf., 2014, 82, 195-215.

15. W. L. Jorgensen, J. Chandrasekhar, J. D. Madura, R. W. Impey and M. L. Klein, J. Chem. Phys., 1983, 79, 926-935.

16. E. Papaleo, P. Mereghetti, P. Fantucci, R. Grandori and L. De Gioia, J Mol Graph Model, 2009, 27, 889-899.

17. J. S. Hub, B. L. de Groot and D. van der Spoel, J. Chem. Theory Comput., 2010, 6, 3713-3720.

18. S. T. Ngo, J. Comput Chem, 2021, 42, 117-123.

19. S. T. Ngo, K. B. Vu, L. M. Bui and V. V. Vu, ACS Omega, 2019, 4, 3887-3893.

20. T. H. Nguyen, G. Rossetti, F. Arnesano, E. Ippoliti, G. Natile and P. Carloni, J. Chem. Theor. Compt, 2014, 10, 35783584. 